\title{
Control and trajectory tracking by flatness of a time-variant stator flux motor
}

\author{
F. Rotella, M. Ayadi, F.J. Carrillo \\ LGP-ENIT, 47 av. d'Azereix, BP48, \\ 65016 Tarbes CEDEX, France. \\ \{rotella, ayadi, carrillo\}@enit.fr
}

\begin{abstract}
By the use of flatness the problem of pole placement, which consists in imposing closed loop system dynamics can be related to tracking. This point of view has been used to enlighten the design of RST controllers in the linear system case with constant parameters. We will see in the present paper that in the case of time-varying linear systems this point of view can also be adopted and leads to design a feedback in terms of the flat output and its derivatives. To implement this feedback a constant gain reduced order observer can be used. The observer poles are also chosen with respect to the trajectory to follow. All these developements are exemplified on a DC motor with a time-variant stator flux, and lead to a simple but powerfull control for tracking a given trajectory for the angular velocity of the motor.
\end{abstract}

\section{Introduction}

For finite-dimensional and fixed linear parameters systems, a well known control design technique is constituted by polynomial two-degrees-of-freedom controllers $[2,8,14]$ which have been introduced forty years ago by Horowitz [10]. Whatever the chosen design method, this powerful method is based on pole placement and presents two deficiencies: it supposes a constant parameter linear model and it needs to know a priori where to place all the poles of the closed-loop system. Following [1]: "the key issue is to choose the closed loop poles. This choice requires considerable insight...". For constant parameter systems, the second drawback of the aforementioned design technique has been overcome in $[18,19]$ by considering the use of a now very well known method for system control design, namely with a flatness point of view. Following [7], flatness is a very interesting property of processes to design a control, specially for trajectory planning and tracking for nonlinear systems but it can also be considered with profit for linear systems. In [18], it has been shown that the closed loop poles choice is enlightened with respect to the trajectory to follow and a clear guideline for polynomial pole placement design can be given. This point of view has been extended to discrete-time systems in [19].

We will see in the present paper that the same benefits can be obtained in the case of linear time-varying parameter models and this will be exemplified on a separated excitation DC motor with a variable stator flux.

The paper is organized as follows. The section 2 is devoted to quickly survey the flatness property and the design control implied for a flat system. In the section 3, the previous methodology is applied on a linear time-varying parameter model. The design and implementation of the controller is performed in two steps. The first one concerns the tracking problem and the second one is with respect to the design of an observer. We will see that a constant gain reduced order Luenberger observer allows to construct all the needed informations, namely the flat output and its derivatives. The proposed design is illustrated in the section 4 .

In the following, we will develop the paper in a continuous-time formulation, for $n \in \mathbb{N}$, we will use the following notations, $u^{(n)}(t)=\frac{d^{n} u(t)}{d t^{n}}$, and for the sake of shortness, for SISO linear systems, but all the results could be adapted to MIMO linear systems.

\section{Short survey on flatness}

The flatness property, which has been introduced recently (1992) $[5,6,7]$ for continuous-time nonlinear systems, leads to interesting points of view for control design. In the following, we will give a short review about flatness of systems and the application of this property to design a controller. The interested reader may find more details in the quoted literature and the references therein.

A system described by:

$$
\dot{X}=f(X, u)
$$

where $X$ is the state vector of dimension $n, u$ is the control vector, possesses the flatness property (or is 
flat) if there exists a vector $z$ :

$$
z=h\left(X, u, u^{(1)}, \ldots, u^{(m)}\right),
$$

where $m \in \mathbb{N}$, such that the components of $z$ are differentially independent, and such that there exist two functions $\mathbf{A}($.$) and \mathbf{B}($.$) , and an integer l$ such that:

$$
\begin{aligned}
X & =\mathbf{A}\left(z, \ldots, z^{(l)}\right), \\
u & =\mathbf{B}\left(z, \ldots, z^{(l+1)}\right) .
\end{aligned}
$$

The selected output $z$ is called a flat output and, obviously, there is no uniqueness. But, as it has been observed on numerous examples, the flat output has a simple and physical meaning.

Roughly speaking, the implications of flatness are of very importance in several ways for control:

- for motion planning: by imposing desired trajectories on the flat output, we can obtain exactly and explicitly the necessary control to generate them, and this without any integration of the differential equations. We can remark that the desired trajectory, $z_{d}(t)$, must be $(l+1)$-times continuously differentiable;

- for linearization: the original system can be transformed into a linear controllable system:

$$
z^{(l+1)}=v
$$

where $v$ is the new input and this by an endogenous feedback, namely, it does not require external variables to be defined. All these developments have been formalized through the Lie-Backlund equivalence of systems in [7] where is given the precise definition of a flat system. Namely a flat system is a system differentially equivalent, through a LieBacklund transformation of trajectories, to a trivial system (5);

- for stabilization: by a linear state feedback:

$$
v=z_{d}^{(l+1)}-\sum_{i=0}^{l} k_{i}\left(z^{(i)}-z_{d}^{(i)}\right)
$$

and a good choice of the $k_{i}$, namely the polynomial $K(p)=p^{i+1}+\sum_{i=0}^{l} k_{i} p^{i}$ is Hurwitz, the trajectory error, with respect to the desired motion $z_{d}(t)$, can be stabilized and goes asymptotically to 0 .

All these points lead to propose a nonlinear feedback which ensures a stabilized tracking of a desired motion for the flat output.

To be more precise the use of flatness leads to the following design for a control. The first step, motion planning, consists in defining a trajectory defined by $z_{d}(t)$, which must be differentiable at the order $(l+1)$, and from (4), the second step is to construct the control:

$$
\begin{aligned}
u= & \mathbf{B}\left(z, \ldots, z^{(l)}\right. \\
& \left.z_{d}^{(l+1)}(t)+\sum_{i=0}^{l} k_{i}\left(z_{d}^{(i)}(t)-z^{(i)}(t)\right)\right), \\
= & \mathbf{H}\left(z, \ldots, z^{(l)}, K(p) z_{d}(t)\right)
\end{aligned}
$$

where the $k_{i}$ coefficients satisfy the stability condition. This last point ensures the motion tracking with respect to the flat output. We must note here that the information needed by this control can be obtained through observers, and a major advantage of this controller with respect to other nonlinear strategies is that it overcomes the problems generated by non stable zeros dynamics [9].

\section{Implication for linear time-varying systems}

Despite the fact that flatness has been firstly developed for nonlinear systems, it has been applied to finitedimensional linear systems [3] and extended for infinitedimensional ones [16]. We will see, in this section, that applying the guideline induced by a flatness based control to a linear time-varying system leads to perform a pole placement in a very natural way.

\subsection{Flatness of linear time-varying systems}

The linear time-varying systems are of great importance due to the fact that these models appear when a local linearization of a nonlinear system is performed around a given trajectory. They have been frequently studied $[12,13,17]$ but some difficulties arise due to the notion of poles and zeros of such systems. Let us consider the system described by the following state space equation:

$$
\begin{aligned}
& \dot{X}(t)=A(t) X(t)+B(t) u(t) \\
& y(t)=C(t) X(t)
\end{aligned}
$$

where $X$ and $u$ have the same meaning than in (1), $y$ is the output and $A(t), B(t)$ and $C(t)$ are time-variant matrices of adapted dimensions, which will be, in all the following, considered as sufficiently differentiable. A non singular, for every $t$, matrix $P(t)$ defines a change of variables:

$$
Z(t)=P(t) X(t)
$$

and leads to the new state space equation:

$$
\begin{aligned}
& \dot{Z}(t)=\bar{A}(t) Z(t)+\bar{B}(t) u(t), \\
& y(t)=\bar{C}(t) Z(t),
\end{aligned}
$$

with $\bar{A}(t)=[P A+\dot{P}] P^{-1}, \bar{B}(t)=P B$ and $\bar{C}(t)=$ $C P^{-1}$. Let us construct the series:

$$
\begin{aligned}
K_{0}(t) & =B(t), \quad \text { for } i=1 \text { to } n \quad: \\
K_{i}(t) & =\dot{K}_{i-1}(t)-A(t) K_{i-1}(t),
\end{aligned}
$$


and the matrix $K(t)$ = $\left[\begin{array}{llll}K_{0}(t) & K_{1}(t) & \cdots & K_{n-1}(t)\end{array}\right]$. Let $\bar{K}(t)$ be the matrix obtained by the same algorithm but from (11), we obtain $\tilde{K}(t)=P(t) K(t)$. When rank $K(t)=n$, for every $t,(9)$ is controllable [21] and we can construct the controllable state form of (9) by the Silverman algorithm [20]. The steps of this algorithm are:

1. Calculation of $\eta(t)=-K^{-1}(t) K_{n}(t)$.

2. Let $F(D)$ the $(n \times n)$ operational matrix defined by:

$$
F(D)=\left[\left\{\begin{array}{ll}
(-1)^{i+j}\left(\begin{array}{l}
j-1 \\
i-1
\end{array}\right) D^{j-i} & \text { if } i \leq j \\
0 & \text { if } i>j
\end{array}\right],\right.
$$

where $D$ stands for the derivative operator $(d / d t)$, we then compute:

$$
\begin{aligned}
\Psi(t) & =F(\mathrm{D}) \eta(t) \\
& =\left[\begin{array}{lll}
\psi_{0}(t) & \cdots & (-1)^{n-1} \psi_{n-1}(t)
\end{array}\right]^{T} .
\end{aligned}
$$

3. Let us consider:

$$
\begin{aligned}
\bar{A}(t) & =\left[\begin{array}{cccc}
0 & 1 & \cdots & 0 \\
\vdots & \ddots & \ddots & \vdots \\
\vdots & & \ddots & 0 \\
0 & \cdots & \cdots & 1 \\
-\psi_{0}(t) & -\psi_{1}(t) & \cdots & -\psi_{n-1}(t)
\end{array}\right], \\
\bar{B} & =\left[\begin{array}{llll}
0 & \cdots & 0 & 1
\end{array}\right]^{T},
\end{aligned}
$$

and the corresponding $\bar{K}(t)$ matrix.

4. $(\bar{A}(t), \bar{B})$ is obtained from $(A(t), B(t))$ by the following transformation matrix:

$$
P(t)=\bar{K}(t) K^{T}(t)\left(K(t) K^{T}(t)\right)^{-1} .
$$

For these new variables, the state space equation can be written as:

$$
\begin{gathered}
\dot{Z}(t)=\bar{A}(t) Z(t)+\bar{B} u(t), \\
y(t)=C(t) P^{-1}(t) Z(t) .
\end{gathered}
$$

If $C(t) P^{-1}(t)=\left[\begin{array}{llll}\gamma_{0}(t) & \gamma_{1}(t) & \cdots & \gamma_{n-1}(t)\end{array}\right]$, and $z_{i}$ denotes the $i$-th component of $Z$, then:

$$
\begin{aligned}
\dot{z}_{1}(t)= & z_{2}(t) \\
& \vdots \\
\dot{z}_{n-1}(t)= & z_{n}(t) \\
\dot{z}_{n}(t)= & u(t)-\sum_{i=0}^{n-1} \psi_{i}(t) z_{i+1}(t), \\
y(t)= & \sum_{i=0}^{n-1} \gamma_{i}(t) z_{i+1}(t),
\end{aligned}
$$

which leads to the differential forms:

$u(t)=z_{1}^{(n)}(t)+\sum_{i=0}^{n-1} \psi_{i}(t) z_{1}^{(i)}(t), \quad y(t)=\sum_{i=0}^{n-1} \gamma_{i}(t) z_{1}^{(i)}(t)$

The variable $z_{1}(t)$, noted $z(t)$, pointed out by the previous relations can be interpreted as the partial state of the system [11] or as a flat output [6].

\subsection{Tracking control}

For a given planned trajectory on the flat output, $z_{d}(t)$, the implemented control will be:

$$
\begin{aligned}
u(t) & =z_{d}^{(n)}(t)+\sum_{i=0}^{n-1} k_{i}\left(z_{d}^{(i)}(t)-z^{(i)}(t)\right)+\psi_{i}(t) z^{(i)}(t) \\
& =z_{d}^{(n)}(t)+\sum_{i=0}^{n-1} k_{i} z_{d}^{(i)}(t)+\sum_{i=0}^{n-1}\left(\psi_{i}(t)-k_{i}\right) z^{(i)}(t) \\
& =K(p) z_{d}(t)+\sum_{i=0}^{n-1}\left(\psi_{i}(t)-k_{i}\right) z^{(i)}(t)
\end{aligned}
$$

where the $k_{i}$ are chosen such that $K(p)$ is a Hurwitz polynomial. The choice is made with respect to the trajectory to track because we get $K(p)\left(z_{d}(t)-z(t)\right)=$ 0 , and we must note here that they are constants. This point of view differs from the usual pole placement for linear time-varying systems obtained by a time-varying state feedback. By denoting:

$$
\psi-k=\left[\begin{array}{c}
\psi_{0}(t)-k_{0} \\
\vdots \\
\psi_{n-1}(t)-k_{n-1}
\end{array}\right],
$$

the previous control can be written as:

$$
u(t)=K(p) z_{d}(t)+(\psi-k)^{T} P(t) X(t) .
$$

\subsection{Observer for $X(t)$}

In order to illustrate the pole placement for our study we will use an usual Luenberger observer but it would be possible to construct a direct observer as it has been suggested in [4] and applied in $[15,18]$ for linear time-invariant systems. Consequently, we want to observe the state of the realization (9). This can be done through the observable form obtained with the Silverman observation algorithm [20]. With:

$$
\begin{aligned}
L_{0}(t) & =C(t), \quad \text { for } i=1 \text { à } n: \\
L_{i}(t) & =\dot{L}_{i-1}(t)+L_{i-1}(t) A(t), \text { and } \\
L^{T}(t) & =\left[\begin{array}{lll}
L_{0}^{T}(t) & \cdots & L_{n-1}^{T}(t)
\end{array}\right],
\end{aligned}
$$

if rank $L(t)=n,(9)$ is observable and we can construct the change of variables, $w(t)=Q(t) X(t)$, such that the system (9) is transformed into the observable form:

$$
\begin{gathered}
\dot{w}(t)=\tilde{A}(t) w(t)+\tilde{B}(t) u(t) \\
y(t)=\left[\begin{array}{llll}
0 & \cdots & 0 & 1
\end{array}\right] w(t)
\end{gathered}
$$


where

$$
\begin{aligned}
& \tilde{A}(t)=\left[\begin{array}{cccc}
0 & \cdots & 0 & -\alpha_{0}(t) \\
1 & \ddots & \vdots & -\alpha_{1}(t) \\
0 & \ddots & 0 & \vdots \\
0 & \cdots & 1 & -\alpha_{n-1}(t)
\end{array}\right], \\
& \tilde{B}(t)=\left[\begin{array}{ccc}
\beta_{0}(t) & \cdots & \beta_{n-1}(t)
\end{array}\right]^{T} .
\end{aligned}
$$

The $\alpha_{i}$ and $\beta_{i}$ are given by the following steps:

1. Calculation of $\rho(t)=-\left[L^{T}(t)\right]^{-1} L_{n}^{T}(t)$.

2. Calculation of:

$$
\alpha(t)=\left[\begin{array}{c}
\alpha_{0}(t) \\
\alpha_{1}(t) \\
\vdots \\
\alpha_{n-1}(t)
\end{array}\right]=[F(\mathrm{D})]^{-1} \rho(t) .
$$

3. With $Q(t)=\left[L^{T}(t) L(t)\right]^{-1} L^{T}(t) \bar{L}(t)^{-1}$, where $\bar{L}(t)$ is the $L(t)$ matrix computed from (15), we get:

$$
Q(t) B(t)=\left[\begin{array}{lll}
\beta_{0}(t) & \cdots & \beta_{n-1}(t)
\end{array}\right]^{T} .
$$

As in (15), the output is the last component, we can use a reduced order observer. Let us group the $n-1$ first components of $w(t)$ in $\chi(t)$ then $^{1}$ :

$$
\dot{\chi}=\left[\begin{array}{cccc}
1 & & & 0 \\
& \ddots & & \vdots \\
& & 1 & 0
\end{array}\right] \chi-\left[\begin{array}{l}
\alpha_{0} \\
\alpha_{1} \\
\vdots \\
\alpha_{n-2}
\end{array}\right] y+\left[\begin{array}{c}
\beta_{0} \\
\beta_{1} \\
\vdots \\
\beta_{n-2}
\end{array}\right] u \text {, }
$$

To overcome the output derivation, we are led to propose the reduced order observer:

$$
\dot{\zeta}=\digamma \zeta+\Theta u+\Gamma y,
$$

$$
\text { where } \zeta(t)=\hat{\chi}(t)-\left[\begin{array}{lll}
\lambda_{0}(t) & \cdots & \lambda_{n-2}(t)
\end{array}\right]^{T} y(t) \text { and }
$$$$
F=\left[\begin{array}{cccc}
1 & & & -\lambda_{0} \\
& \ddots & & -\lambda_{1} \\
& & 1 & -\lambda_{n-2}
\end{array}\right],
$$$$
\Theta=\left[\begin{array}{c}
\beta_{0}-\beta_{n-1} \lambda_{0} \\
\beta_{1}-\beta_{n-1} \lambda_{1} \\
\vdots \\
\beta_{n-2}-\beta_{n-1} \lambda_{n-2}
\end{array}\right] \text {, }
$$$$
\Gamma=\left[\begin{array}{c}
-\alpha_{0}-\dot{\lambda}_{0}+\left(\alpha_{n-1}-\lambda_{n-2}\right) \lambda_{0} \\
\lambda_{0}-\alpha_{1}-\dot{\lambda}_{1}+\left(\alpha_{n-1}-\lambda_{n-2}\right) \lambda_{1} \\
\vdots \\
\lambda_{n-3}-\alpha_{n-2}-\dot{\lambda}_{n-2}+\left(\alpha_{n-1}-\lambda_{n-2}\right) \lambda_{n-2}
\end{array}\right] .
$$

\footnotetext{
${ }^{1}$ For space reasons, we dropped the time argument.
}

As the error dynamics are given by the matrix $F$, we choose for all $i, \lambda_{i}(t)$ as constant parameters to give an asymptotic observer. The poles for this observer are fixed with respect to the trajectory to track on the flat output. The observation of $X(t)$ is obtained by:

$$
\hat{X}(t)=Q^{-1}(t)\left[\zeta(t)+\left[\begin{array}{lll}
\lambda_{0} & \cdots & \lambda_{n-2}
\end{array}\right]^{T} y(t)\right] .
$$

\section{DC motor with time-variant flux}

Let us consider the separated excitation DC motor described by the equations:

$$
\begin{aligned}
L_{s} \frac{d I_{s}(t)}{d t}+R_{s} I_{s}(t) & =V_{s}(t), L_{s} I_{s}(t)=\Phi(t),(16) \\
L_{r} \frac{d I_{r}(t)}{d t}+R_{r} I_{r}(t) & =V_{r}(t)-K_{e} \Phi(t) I_{r}(t), \\
J \frac{d \Omega(t)}{d t}+f \Omega(t) & =K_{m} \Phi(t) I_{r}(t)
\end{aligned}
$$

where the indices $s$ and $r$ denotes respectively the stator and rotor electrical subsystems, and $R_{s}, R_{r}$ the resistances (resp. 0.1 and $2 \Omega$ ), $L_{s}, L_{r}$ the inductances (resp. $10^{-3}$ and $0.5 \mathrm{H}$ ), $I_{s}, I_{r}$ the coil currents, $V_{s}, V_{r}$ the coil voltages, $\Phi(t)$ the flux produced by the stator current, $K_{e}, K_{m}$ the constant back-emf and electromagnetic torque parameters (resp. 0.1 and $0.1 \mathrm{Nm} / \mathrm{Wb} \mathrm{A}), J$ the rotor and load inertias $(0.02$ $\left.\mathrm{kg} / \mathrm{m}^{2} \mathrm{~s}^{2}\right), f$ the damping coefficient $(0.2 \mathrm{Nms})$ and $\Omega$ the rotor velocity. The considered input is $V_{r}$ and the output is $\Omega$. For simplicity sake we will suppose that a variation in the stator coil voltage induces a periodic time-varying flux given by:

$$
\Phi(t)=\Phi_{0}(1+0.1 \sin (\pi t)),
$$

and our objective is to follow a specified trajectory for the rotor velocity.

By elimination of some variables, the following differential equation relates the rotor velocity and the rotor coil voltage:

$$
\ddot{\Omega}+\frac{d}{d t}\left[\alpha_{1}(t) \Omega\right]+\alpha_{0}(t) \Omega=\beta(t) V_{r},
$$

where:

$$
\begin{aligned}
\alpha_{0}(t) & =\frac{K_{m} K_{e}}{J L_{r}} \Phi^{2}-\frac{f \dot{\Phi}}{J \Phi}+\frac{R_{r} f}{L_{r} J}+\frac{\ddot{\Phi} \Phi-\dot{\Phi}^{2}}{\Phi^{2}} \\
\alpha_{1}(t) & =\frac{f}{J}+\frac{R_{r}}{L_{r}}-\frac{\dot{\Phi}(t)}{\Phi(t)} \\
\beta(t) & =\frac{K_{m} \Phi(t)}{J L_{r}}
\end{aligned}
$$

From these relations, we deduce the state space observ- 
able form:

$$
\begin{aligned}
& \dot{X}(t)=\left[\begin{array}{cc}
0 & -\alpha_{0}(t) \\
1 & -\dot{\alpha}_{1}(t)
\end{array}\right] X(t)+\left[\begin{array}{c}
\beta(t) \\
0
\end{array}\right] V_{r}(t) \\
& \Omega(t)=\left[\begin{array}{cc}
0 & 1
\end{array}\right] X(t)
\end{aligned}
$$

In order to construct the flat output, we must obtain the controllable form of these equations, and the Silverman algorithm gives:

$$
\begin{aligned}
& \dot{Z}(t)=\left[\begin{array}{cc}
0 & 1 \\
-\psi_{0}(t) & -\psi_{1}(t)
\end{array}\right] Z(t)+\left[\begin{array}{l}
0 \\
1
\end{array}\right] V_{r}, \\
& \Omega(t)=\left[\begin{array}{cc}
K_{m} L_{r} \Phi(t) & 0
\end{array}\right] Z(t),
\end{aligned}
$$

where $Z(t)=P(t) X(t)$, and:

$$
P(t)=\frac{1}{K_{m} \Phi(t)}\left[\begin{array}{cc}
0 & J L_{r} \\
J L_{r} & -\left(f L_{r}+R_{r} J\right)
\end{array}\right] .
$$

The $\psi_{0}(t)$ and $\psi_{1}(t)$ expressions are given by:

$$
\begin{aligned}
& \psi_{0}(t)=\frac{1}{\beta(t)}\left(\ddot{\beta}(t)+\alpha_{1}(t) \dot{\beta}(t)\right)+\alpha_{0}(t)+\dot{\alpha}_{1}(t), \\
& \psi_{1}(t)=\frac{1}{\beta(t)}\left(2 \dot{\beta}(t)+\alpha_{1}(t) \beta(t)\right) .
\end{aligned}
$$

From the previous part, the first component of $Z(t)$, namely $z(t)$, is a flat output for this system, which leads to the following controller:

$$
\begin{aligned}
V_{r}(t)= & \ddot{z}_{d}(t)+k_{1}\left(\dot{z}_{d}(t)-\dot{\hat{z}}(t)\right)+k_{0}\left(z_{d}(t)-\hat{z}(t)\right) \\
& +\psi_{1}(t) \dot{\hat{z}}(t)+\psi_{0}(t) \hat{z}(t),
\end{aligned}
$$

where $K(p)=p^{2}+k_{1} p+k_{0}$ is Hurwitz and defines the dynamics for the tracking error.

To observe $Z(t)$, from the previous equations, we only want to estimate the first component of $X(t)$. With $\xi(t)=\hat{x}_{1}(t)-L y(t)$, we propose the following observer:

$\dot{\xi}(t)=-L \xi(t)+\beta(t) V_{r}+\left(-\alpha_{0}(t)+\alpha_{1}(t) L-L^{2}(t)\right) y(t)$,

where $L$ is a constant giving the observer pole. From $\hat{X}(t)=\left[\begin{array}{ll}\xi(t)+L y(t) & y(t)\end{array}\right]^{T}$, we obtain $\hat{Z}(t)$ with $\hat{Z}(t)=P(t) \hat{X}(t)$, which leads to the controller:

$V_{r}(t)=K(p) z_{d}(t)-\left[\begin{array}{ll}k_{0}-\psi_{0}(t) & k_{1}-\psi_{1}(t)\end{array}\right] P(t) \hat{X}(t)$.

Let us define the following trajectory for the flat output, $10 z^{d}(t)-1=$

$$
\begin{cases}0 & \text { if } t \leq t_{0} \\ r(t) & \text { if } t_{0} \leq t \leq t_{f} \\ 1 & \text { if } t \geq t_{f}\end{cases}
$$

with $t_{0}=2 \mathrm{sec}$ and $t_{f}=3 \mathrm{sec}$ and:

$r(t)=21\left(\frac{t-t_{0}}{t_{f}-t_{0}}\right)^{5}-35\left(\frac{t-t_{0}}{t_{f}-t_{0}}\right)^{6}+15\left(\frac{t-t_{0}}{t_{f}-t_{0}}\right)^{7}$.
For this trajectory, the dynamics for the tracking error are chosen to have a second order dynamics with $\xi=0.7$ and $\omega_{n}=6 \mathrm{rad} / \mathrm{s}$ which correspond to the following closed loop poles $-4.20 \pm 4.28 i$ and the observer pole is chosen as -15 . The simulation results are displayed in the figure 1 and show the effectiveness of the proposed strategy.
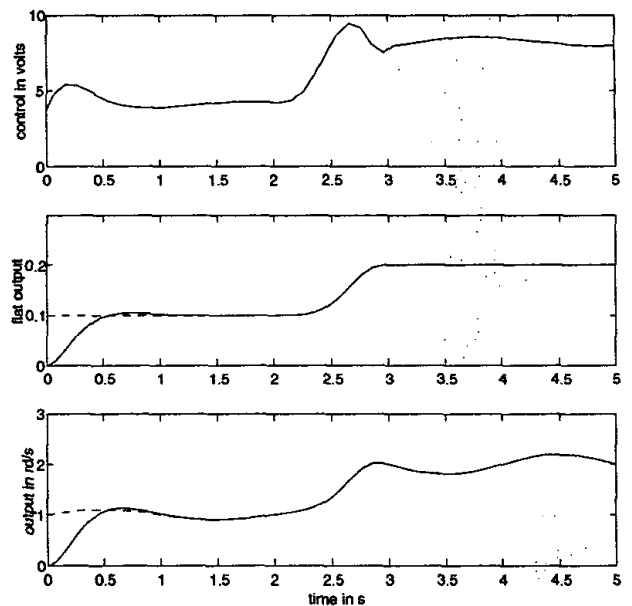

Figure 1: Flatness control with a reduced order observer.

If we want now to follow a trajectory for the rotor velocity, as we have $\Omega(t)=\frac{K_{m} L_{r}}{J} \Phi(t) z(t)$, we can get:

$$
z_{d}(t)=\gamma(t) \Omega_{d}(t)
$$

with $\gamma(t)=\left(\frac{K_{m} L_{x}}{J} \Phi(t)\right)^{-1}$. If we want a constant rotor velocity $\Omega_{d}(t)=\Omega_{d}$, we get $\dot{z}_{d}(t)=\Omega_{d} \dot{\gamma}(t)$ and $\ddot{z}_{d}(t)=\Omega_{d} \ddot{\gamma}(t)$. With $\Omega_{d}=1 \mathrm{rad} / \mathrm{s}$, we obtain the simulation results, for the same performances and parameter choice than previously, displayed in the figure 2.

\section{Conclusion}

In this paper we have underlined that the use of a flatness point of view allows a simplification in the design of a pole placement control for linear time-varying parameter systems. The main feature of the flatness approach is to avoid the problem of the closed loop poles choice which are constituted of the observer poles and those obtained with a state feedback [2]. Now the design is focused in the choice of the trajectory $z_{d}$ to follow and the tracking dynamics with $K(p)$. Moreover, the use of a reduced order observer allows to choose constant gains for the observer poles. It is also possible to use a direct observer [4] but, for space limitation, the detailed computations and the simulation results have 

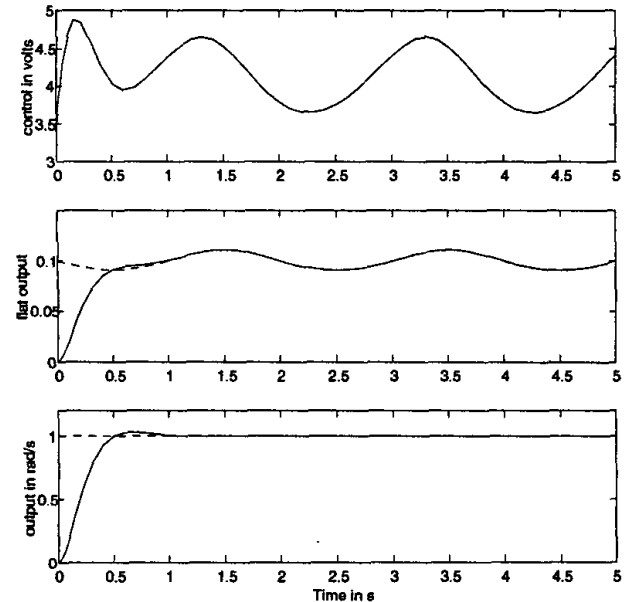

Figure 2: Rotor velocity tracking with a reduced order observer.

not been included here. These developments were done in a continuous-time framework, but are transposable for discrete-time systems. For sake of simplicity we have also developed the method in a single input single output framework, but it could remain valid in the multivariable case. The proposed method has been exemplified on the trajectory tracking of a DC motor where the stator flux was time-varying. A direction of future works will be to attack the problem of the numerical implementation of such controllers and to tackle with the adaptive implementation of the proposed control to overcome uncertainties in model parameters.

\section{References}

(1] Aström, K.J., Bernardson, B., Ringdhal, A., "Solution using robust adaptive pole placement", $E C C^{\prime} 91$, Grenoble, pp.2341-2346, 1991.

[2] Aström, K.J., Wittenmark, B., Computer controlled systems, Theory and design, Prentice Hall, 1990.

[3] Bitaud, L., Fliess, M., Lévine, J., "A flatness based control synthesis of linear systems and application to windshield wipers", ECC'97, Brussels, 1997.

[4] Fliess, M., "Sur des pensers nouveaux faisons des vers anciens", CIFA2000, Lille, pp. 26-36, July, 2000.

[5] Fliess, M., Lévine, J., Martin, Ph, Rouchon, P., "On differentially flat nonlinear systems", IFAC-Symp. NOLCOS'92, Bordeaux, pp. 408-412, 1992.

[6] Fliess, M., Lévine, J., Martin, Ph, Rouchon, P., "Flatness and defect of nonlinear systems: introductory theory and applications", Int. J. Control, vol. 61, n. 6, pp. 1327-61, 1995.

[7] Fliess, M., Lévine, J., Martin, Ph, Rouchon, P.,
"A Lie-Bäcklund approach to equivalence and flatness of nonlinear system", IEEE Trans. Aut. Control, vol. 44, n. 5, pp. 922-937, 1999.

[8] Franklin, G.F., Powell, J.D., Workman, M., Digital control of dynamic systems, Addison-Wesley, 1998.

[9] Isidori, A., Nonlinear control systems, SpringerVerlag, 1989.

[10] Horowitz, I.M., Synthesis of feedback systems, 1963.

[11] Kailath, T., Linear systems, Prentice-Hall, 1980.

[12] Kalman, R.E., "A new approach to linear filtering and prediction problems", Trans. ASME ser. $D, J$. Basic Engineering, vol. 82, pp. 35-45, 1960.

[13] Kamen, E.W., "Fundamentals of linear timevarying systems", chap. 25, The control handbook, Levine W.S. (Ed.), pp. 451-468, CRC Press, 1996.

[14] Kucera, V., Analysis and design of discrete linear control systems, Prentice Hall, 1991.

[15] Marquez, R., Delaleau, E., Fliess, M., "Commande par PID généralisé d'un moteur électrique sans capteur mécanique", CIFA2000, Lille, pp. 453-458, July, 2000.

[16] Mounier, H., Rudolph, J., "Extending flatness to infinite dimensional systems", to appear.

[17] Richards, J.A., Analysis of periodically timevarying systems, Springer-Verlag, 1983.

[18] Rotella, F., Carrillo, F.J., Ayadi, M., "Polynomial controller design based on flatness", IFAC-Symp. on System Structure and Control, Prague, 2001.

[19] Rotella, F., Carrillo, F.J., Ayadi, M., "Digital flatness-based robust controller applied to a thermal process", IEEE Conference on Control Applications, Mexico, 2001.

[20] Silverman, L.M., "Transformation of timevariable systems to canonical (phase-variable) form", IEEE Trans. Aut. Control, AC-11, pp. 303-306, 1966.

[21] Silverman, L.M., Meadows, H.E., "Controllability and observability in time-variable linear systems", SIAM J. Control and Optimization, n. 5, pp. 64-73, 1967. 